\title{
Direct Coupling of Power Active Filters with Photovoltaic Generation Systems with Improved MPPT Capability
}

\author{
G. Grandi, Member, IEEE, D. Casadei, Member, IEEE, C. Rossi
}

\begin{abstract}
Photovoltaic sources can be usefully combined with power active filters in order to regulate both the active and the reactive power injected into the mains. In this way, the system is able to compensate undesired load characteristics such as phase unbalances, power flickers, and low-order current harmonics, improving the power quality. In order to avoid an intermediate dc-dc chopper, a direct coupling between the PV panels and the dc-link of the voltage source inverter is considered in this paper. The dc-link voltage is continuously regulated between upper and lower limits for tracking the maximum power point of the $P V$ panels. The proposed MPPT algorithm is based on real-time processing of current and voltage ripple of the PV source that is generated by the switching operation of the PWM inverter. The system performance is investigated emphasizing the transient behavior in presence of sudden variations of the solar irradiance. The theoretical developments are verified by realistic numerical simulations.
\end{abstract}

Index terms - Active filters, Photovoltaic cells, Power conditioning, Power quality, Solar power generation.

\section{INTRODUCTION}

$\mathrm{P}$ HOTOVOLTAIC energy seems to become one of the most important renewable energy resources in the near future, since it is clean, pollution free, and inexhaustible.

Due to the rapid growth in semiconductors and power electronic techniques, the solar energy is of increasing interest in electrical power applications, and a large research activity has been carried out in this field over the last years.

Nowadays, despite of the known advantages, the installation cost is still high and the PV energy is not competitive yet with respect to the traditional fossil-based energy resources.

Since PV panels have relatively low conversion efficiency, minimizing the losses in the power electronic converters, and maximizing the electric power extracted from the PV source can substantially reduce the whole system cost. On the basis of these considerations, in this paper the analysis is focused on a system topology with a direct coupling between the PV panels and the voltage source inverter (VSI) employed to inject the solar power into the utility grid. A block diagram of the corresponding PV generation system is represented in Fig. 1. In this way, the need of an intermediate dc-dc chopper is avoided, increasing the efficiency and reducing the cost of the overall system.

In order to extract the maximum power from the PV source this simplified structure, the dc-link voltage must be regulated according with a maximum power point tracking algorithm (MPPT), which directly acts on the control system of the VSI. In this case, the dc-link voltage excursion should be limited

G. Grandi, D. Casadei, C. Rossi are with the Department of Electrical Engineering, University of Bologna, via Risorgimento 2, I-40136 Bologna ITALY. (e-mail: elettro@mail.ing.unibo.it). within a fixed range, to ensure correct power injection into the utility grid (lower limit), and to satisfy the voltage insulation requirements of the power electronic components (upper limit).

An improved MPPT algorithm is presented in this paper. It is based on the analogue processing of current and voltage ripple of the PV panels, which is generated by the switching operation of the PWM inverter. The advantages of the method proposed in this paper are emphasized with respect to the traditional ones, such as the "perturbation and observation" method and the "incremental conductance" method.

The VSI can be controlled such as an active filter, contributing to satisfy the power quality requirements of the utility grid. In this way, the system is able to deliver besides the solar power, reactive power and current harmonics in order to compensate non-linear and/or unbalanced loads [1]. The control strategy is based on keeping the source currents in phase with the corresponding positive sequence components of the supply voltages. This method leads to sinusoidal balanced source currents, regardless to source voltage unbalance or distortion [2].

Numerical simulations are presented for verifying the system performance in the case of sudden variations of the solar irradiance and/or the load power.

\section{MPPT ALGORITHM}

The goal of MPPT algorithms is to extract the maximum power from the PV panels. Usually, the condition $\partial p / \partial v=0$ is adopted to locate this operating point, since PV panels show a unique global maximum point.

\section{A. Background and Proposed MPPT Algorithm}

As it is known, the $i=i(v)$ characteristic of PV panel is affected by environmental variables, i.e., solar irradiance $\left(I_{i r r}\right)$ and temperature $(\theta)$. In particular, the short-circuit current is approximately a linear function of the illumination intensity,

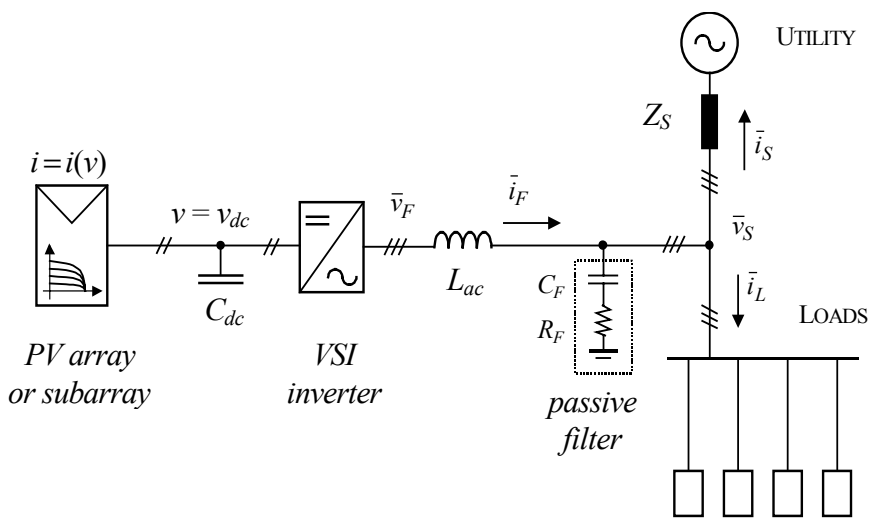

Fig. 1. Block diagram of the PV generation system 


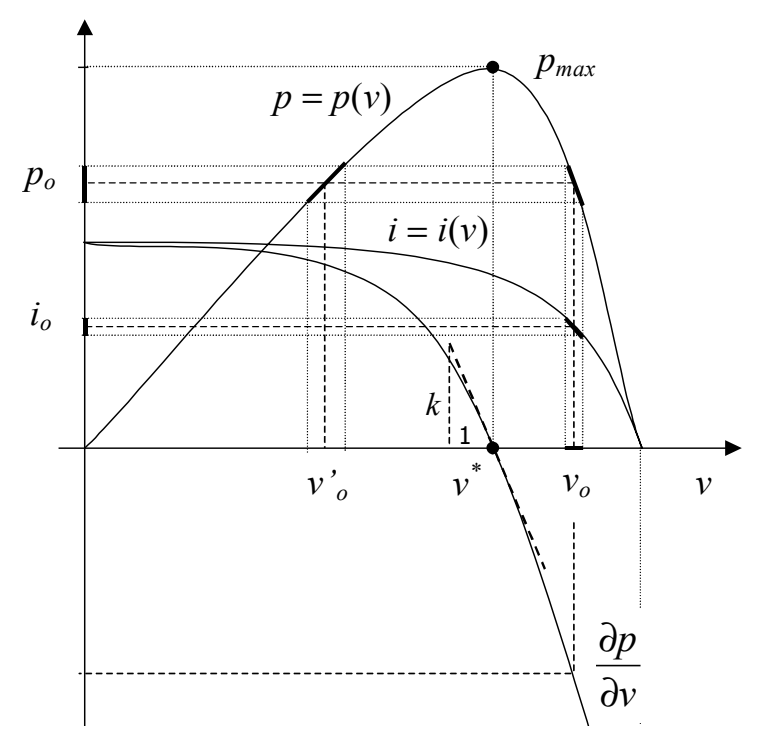

Fig. 2. PV current, power, and power slope characteristics vs. voltage

and the temperature changes the open-circuit voltage of about $-0.4 \% /{ }^{\circ} \mathrm{C}$. For these reasons, static settings such as constant voltage, constant current, or even constant impedance, are not effective to locate the MPP under both types of variation. Then, in order to maximize the power extracted from the PV panels, dynamic methods have been largely investigated.

In the "perturbation and observation" method, the controller perturbs the operating point and observes the resulting $\Delta P$ and $\Delta V$ to identify the actual operating region. Then, the reference voltage is increased or decreased according to these measured quantities. The implementation of this method is simple but it does not allow the tracking of rapid change in environmental conditions.

In the "incremental inductance" method, the MPP is tracked by comparing the incremental conductance and the instantaneous conductance of the PV panels, according with the condition $\partial p / \partial v=0$ :

$$
\frac{\partial p}{\partial v}=i+v \frac{\partial i}{\partial v}=0 \Rightarrow \frac{\partial i}{\partial v}=-\frac{i}{v} .
$$

Some variants to these methods have been considered in order to improve the dynamic performance and/or to reduce undesired oscillations around the MPP [3], [4], [5].

The proposed MPPT algorithm is based on the determination of the output power slope of the PV panels versus voltage, i.e., the power derivative $\partial p / \partial v$. This quantity is utilized as representative of the "voltage error", i.e., the difference between the actual voltage of the PV panels and the voltage $v^{*}$ corresponding to the MPP. The qualitative behavior of $\partial p / \partial v$ is represented tin Fig. 2: in the region near to $v^{*}$ it can be considered a straight line having the slope $k$.

Since the PV panels are directly connected to a VSI operating in switching mode, a switching ripple will affect the PV output voltage and current, for any operating condition of the VSI. The amplitude of the ripple is an inverse function of the dc-link capacitor size, $C_{d c}$, and of the switching frequency $f_{c}$ (PWM carrier frequency). Then, the ripple can be considered itself as an embedded dynamic test signal useful to determine $\partial p / \partial \nu$. A similar approach has been introduced in [6] and [7] to estimate the incremental conductance. A method based on the ripple analysis, called "ripple correlation control", useful to exploit gradient information for optimization purposes is described in [8].

In order to derive the proposed MPPT algorithm, let us to consider a generic periodic function $x(t)$ having the moving average component $\bar{x}(t)$ over the period $T$, and the alternative component $\tilde{x}(t)$, i.e., a ripple, respectively defined as:

$\bar{x}(t)=\frac{1}{T} \int_{t-T}^{t} x(\tau) d \tau$,

$\widetilde{x}(t)=x(t)-\bar{x}(t)$.

Applying these definitions to the output voltage and power of the PV panels, and considering as period $T=1 / f_{c}$, leads to

$v(t)=\bar{v}(t)+\widetilde{v}(t)$,

$p(t)=\bar{p}(t)+\widetilde{p}(t)$.

The average operating point $\left(v_{o}, \mathrm{p}_{o}\right)$ on the $p=p(v)$ characteristic, and the corresponding voltage and power alternative components are represented in Fig. 2, according to

$\bar{p}(t)=p(\bar{v}(t))=p\left(v_{o}\right)=p_{o}, \quad v_{o}=\bar{v}(t)$.

Assuming the curve $p=p(v)$ still valid for dynamic analysis [6] and linearizing nearby the average operating point leads to a simple correlation between the power ripple and the voltage ripple, expressed as

$\widetilde{p}(t) \cong\left(\frac{\partial p}{\partial v}\right)_{v_{o}} \widetilde{v}(t)$.

The power derivative vs. voltage can be calculated by (7) as a function of power and voltage ripple for the given operating point $v_{o}$. In [9] this power derivative is expressed as the time derivative ratio $\dot{p} / \dot{v}$ and approximated by a sign function to extract its sign only, in the same way as in the "perturbation and observation" method.

In this paper we propose to avoid calculations based on instantaneous values, considering instead of (7) the moving average of the product of $(7)$ and $\widetilde{v}(t)$, leading to

$$
\int_{t-T}^{t} \widetilde{p}(\tau) \widetilde{v}(\tau) d \tau \cong \int_{t-T}^{t}\left(\frac{\partial p}{\partial v}\right)_{v_{o}} \widetilde{v}^{2}(\tau) d \tau
$$

Then, the power derivative can be evaluated as the following ratio

$$
\left(\frac{\partial p}{\partial v}\right)_{v_{o}} \cong \frac{\int_{t-T}^{t} \tilde{p}(\tau) \widetilde{v}(\tau) d \tau}{\int_{t-T}^{t} \tilde{v}^{2}(\tau) d \tau}
$$

The voltage and power alternative components utilized in (9) can be calculated on the basis of (3) and (2), leading to 


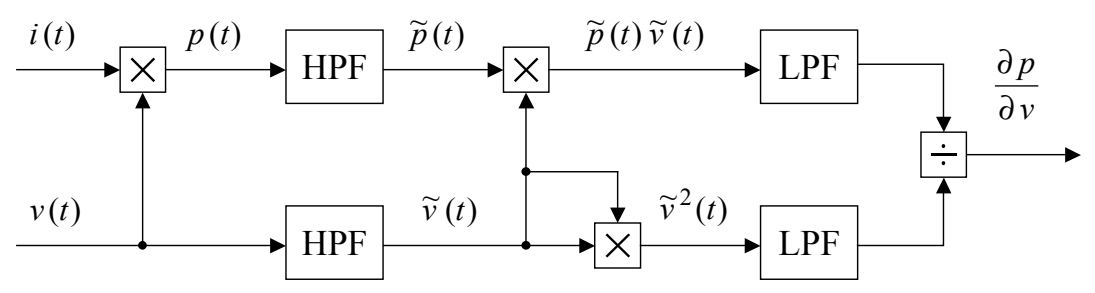

Fig. 3. Estimation of the PV power derivative by using filtering blocks

$$
\begin{gathered}
\widetilde{v}(t)=v(t)-\frac{1}{T} \int_{t-T}^{t} v(\tau) d \tau \\
\tilde{p}(t)=p(t)-\frac{1}{T} \int_{t-T}^{t} p(\tau) d \tau
\end{gathered}
$$

Alternatively, a more traditional filtering approach can be adopted to determine the power slope [7]. In particular, highpass filters (HPF) can be used to extract the alternative components instead of (10) and (11). In the same way, low-pass filters (LPF) can be used for averaging the ripple products instead of the integrals in (9). The corresponding block diagram is depicted in Fig. 3.

\section{B. Voltage Regulation of the PV panels}

Usually, dc-link voltage controllers perform the regulation by acting on the instantaneous error between a reference voltage and the actual voltage. Since the characteristic $i=i(v)$ of the PV panels always has a negative slope, the voltage can be increased by decreasing the output current, and vice versa. In this case, the reference voltage is represented by the voltage $v^{*}$ corresponding to the MPP for the given environmental conditions (see Fig. 2).

As shown in Fig. 4 (left side), the power derivative $\partial p / \partial v$ is utilized as representative of the voltage error $\Delta v=v^{*}-v$, being almost linear the relationship between these two variables in the region around the MPP. The proportional coefficient $k$ is introduced in Fig. 2; it is a function of the solar irradiance intensity $I_{\text {irr }}$ and the temperature $\theta$ of the PV panels. Since the regulation loop contains a regulator $\left(R_{1}(s)\right.$ in Fig. 4), a constant value can be assumed for $k$. The output of the regulator is the reference current for the grid inverter.

The system behavior can be easily described as follows: when the estimated power derivative is positive, the operating point is on the left of the MPP, and the regulator should decrease the current extracted from the PV panels. The converse occurs when the estimated power derivative is negative: the operating point is on the right of the MPP, and the regulator increases the current extracted from the PV panels. Finally, when the power derivative approaches zero it means that the MPP is reached, and the output current of the PV panels is kept constant by the regulator.

A correct behavior of the VSI connecting the PV panels to the grid is obtained if the excursion of the dc-link voltage is limited within a fixed range. The dc-link voltage should be high enough to ensure correct power injection into the utility grid (lower limit). On the other hand, it should satisfy the voltage insulation requirements of the power electronic components (upper limit). For this reason, it is useful to estimate the reference voltage $v^{*}$ and to pass it through a limiter, as shown in the inner dashed rectangle of Fig. 4.

\section{VSI CONTROLLER}

The aim of the power conditioner is to regulate the dc-link voltage $v_{d c}$ in order to track the MPP of the PV modules, being $v_{d c}=v_{P V}$. The control is indirectly performed by regulating the current extracted from the PV panels and injected into the power grid (source current, $\bar{i}_{S}$ ), according to the block diagram shown in Fig. 5. In this way, all the power coming from the PV modules is transferred to the electric network.

In particular, the desired amplitude of the source currents, $I_{S}^{*}$, is generated by the regulator $R(s)$, considering the estimated PV power derivative $\partial p / \partial v$ as input variable. Note that the regulator block $R(s)$ shown in Fig 5 include the scheme represented inside the outer rectangle of Fig. 4.

The reference value of the instantaneous source current vector, $\bar{i}_{S}^{*}$, is generated on the basis of the magnitude $I_{S}^{*}$, and the phase angle of the positive sequence component of the supply voltages [2], which is represented by the unity space vector $\hat{v}_{S}^{+1}$ in Fig. 5 .

The proposed structure allows the system to be able to compensate non-linear, reactive, pulsating, and/or unbalanced loads [1]. In this case, the ac current controller requires the measurement of both load and inverter currents, $\bar{i}_{L}$ and $\bar{i}_{F}$ (see Fig. 5). The load current is used to calculate the reference value of the inverter current $\bar{i}_{F}^{*}$, on the basis of the reference source current $\bar{i}_{S}^{*}$, given by the regulator $R(s),\left(\bar{i}_{F}^{*}=\bar{i}_{S}^{*}+\bar{i}_{L}\right)$.

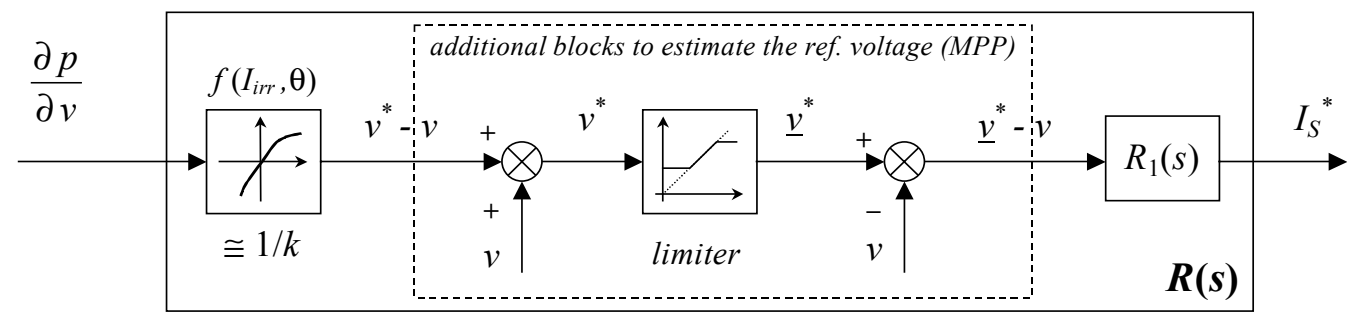

Fig. 4. Generation of voltage and current references 


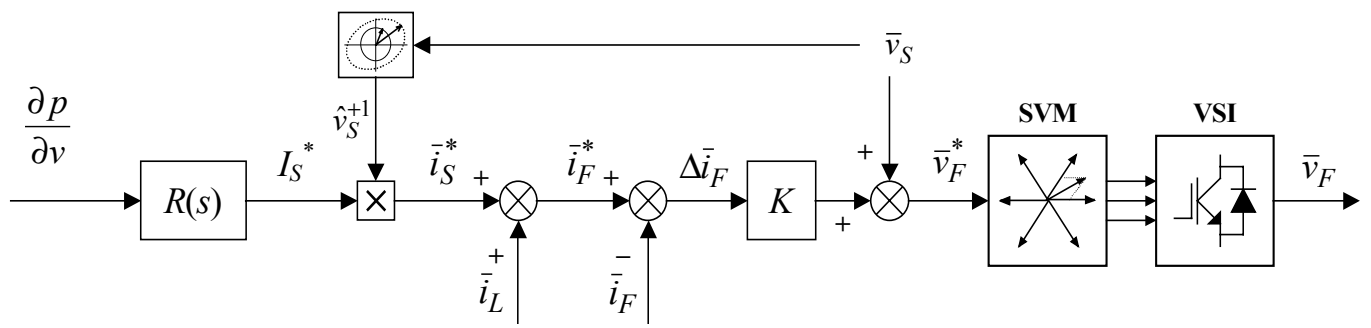

Fig. 5. Proposed regulation scheme for the VSI controller

The measurement of the inverter current is used to implement the ac current control loop. A hysteresis current regulator acting on the instantaneous current error $\Delta \bar{i}_{F}=\bar{i}_{F}^{*}-\bar{i}_{F}$ can be employed to determine the inverter switch states. Alternatively, a PWM (or SVM) current regulator can be used avoiding the drawbacks of hysteresis current controllers. In this case, the reference voltage for the inverter can be calculated by the voltage equation written across the ac-link inductance $L_{a c}$, according with the block diagram represented in Fig. 1. Neglecting the resistive effects and introducing a variational model, the voltage equation yields

$$
\bar{v}_{F}^{*}=\bar{v}_{S}+\frac{L_{a c}}{\Delta t} \Delta \bar{i}_{F} \text {. }
$$

The parameter $L_{a c} / \Delta t$ in (12) can be adjusted to obtain the desired regulator performance.

\section{SYSTEM PERFORMANCE}

The proposed control scheme, represented in the block diagrams of Figs. 4 and 5, has been implemented considering a three-phase, single-stage converter, as represented in Fig. 1. The whole power conversion system has been numerically simulated by the Simulink environment of Matlab.

The PV panels have been electrically represented by the well-known single-exponential model [10], fitted on the $I-V$ characteristic of a series/parallel arrangement of Solar Shell SP150 modules. The main characteristics of the PV generation system are summarized in Table I. The performance of the power conditioning system connected to the PV array has been evaluated both in steady state and transient operating conditions determined by start-up and solar irradiance variations.

Figs. 6 and 7 show the time behavior of the power and voltage alternative components, $\widetilde{p}$ and $\widetilde{v}$, which are evaluated in the MPPT algorithm and are used in computation of the power derivative. In particular, Fig. 6 is referred to an operating point on the left of the MPP, i.e., $v_{d c}<v^{*}$. Note that in this case $\widetilde{v}$ and $\widetilde{p}$ are in phase agreement. Fig 7 is referred to an operating point on the right of the MPP, i.e., for $v_{d c}>v^{*}$. Note that in this case $\widetilde{v}$ and $\widetilde{p}$ are in phase opposition. For all the operating conditions the frequency of oscillation of $\widetilde{v}$ and $\widetilde{p}$ is always related to the switching frequency $\left(f_{s w}=10\right.$ $\mathrm{kHz})$.

Fig. 8 shows the steady-state waveforms of source voltage and source current (utility grid). As expected, the source current is sinusoidal and in phase agreement with the corresponding line to neutral voltage.

Fig. 9 shows the time behavior of the signal representing the calculated power derivative $\partial p / \partial v$ around the maximum power point. This figure is taken by varying the dc-link voltage reference in the range $v^{*} \pm 7 \mathrm{~V}$, with a constant slope. As predicted for this operating region, the calculated $\partial p / \partial v$ is linear and equals to zero exactly in the point of maximum power $\left(v_{d c}=v^{*}\right)$.

Figs. 10-12 show the performance of the PV generation system in tracking the maximum power point of the PV panels during a transient of solar irradiance. From the starting operating point (1), the system reaches the MPP in (2). Then, as a consequence of a $50 \%$ reduction in the solar irradiance, the operating point moves to the new MPP in (3). Fig. 10 and 11 represent the system characteristics respectively on the $I-V$ and $P$ - $V$ diagram. Fig. 12 shows the time behavior of the PV voltage and current during the seeking of the new MPP. It can be observed that the time employed by the MPPT algorithm to reach the new maximum power point is about $50 \mathrm{~ms}$. Once the new MPP is reached, only very small oscillations persist around the MPP.

Fig. 13 shows the waveforms of the source voltage and current, during the transient of solar irradiance. After the irradiance change, the source current rapidly changes its amplitude and remains sinusoidal and in phase agreement with the corresponding line to neutral source voltage.

\begin{tabular}{|l|r|r|l|r|}
\hline \multicolumn{5}{|c|}{ TABLE I } \\
\hline MaIN ChARACTERISTICS OF THE PV GENERATION SYSTEM
\end{tabular}




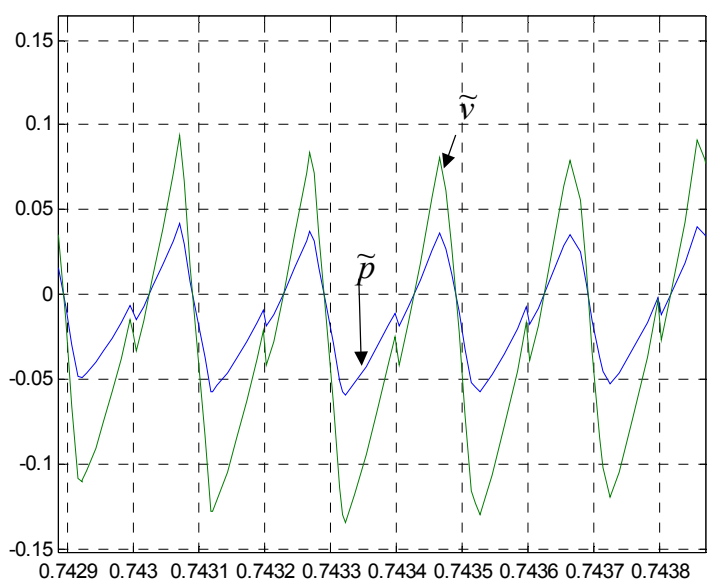

Fig. 6. Voltage and power alternative components for $v<v^{*}$

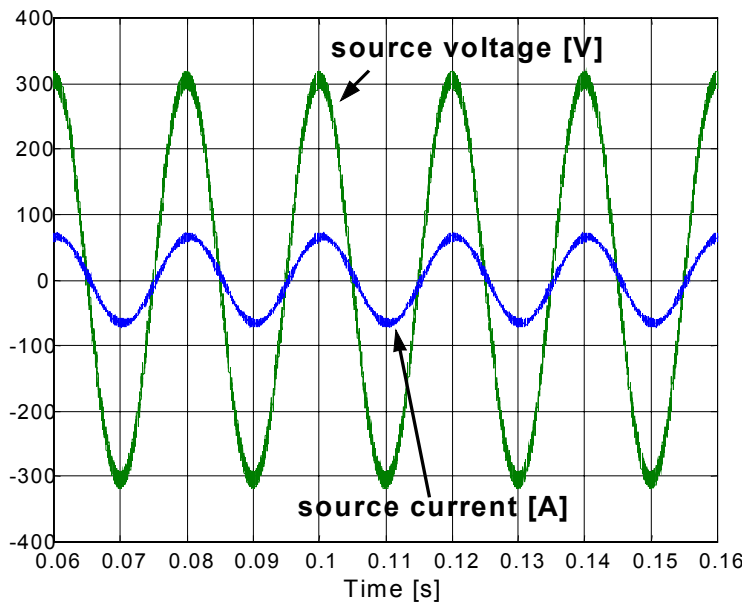

Fig. 8. Source voltage and current in steady-state condition

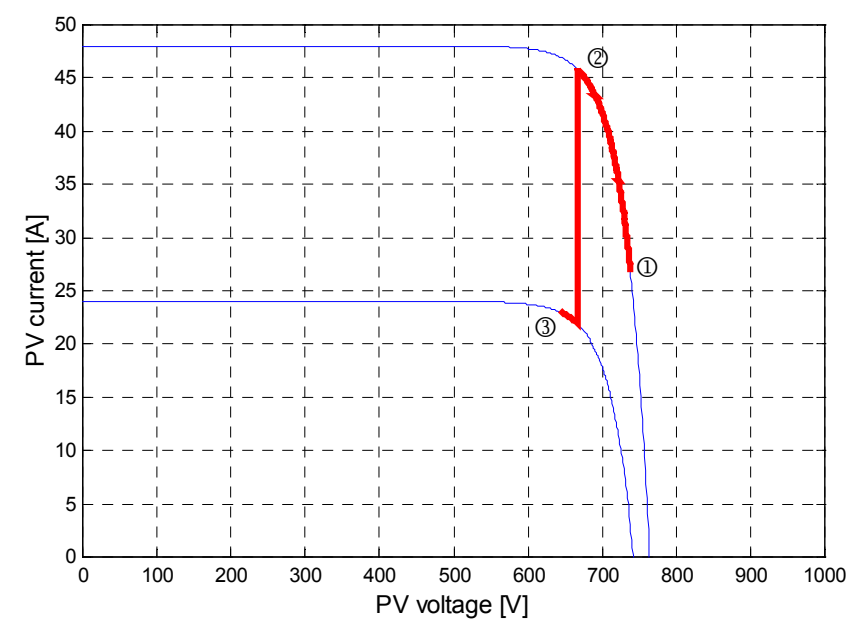

Fig. 10. Effect of a $-50 \%$ solar irradiance transient on the $I-V$ diagram

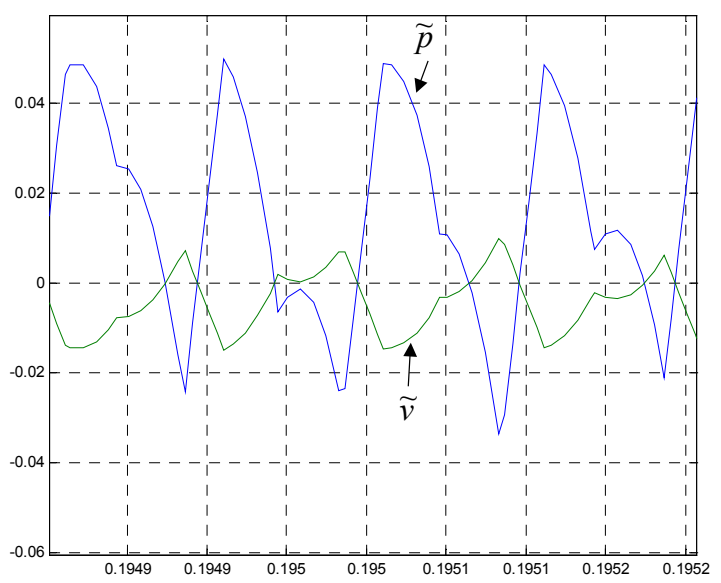

Fig. 7. Voltage and power alternative components for $v>v^{*}$
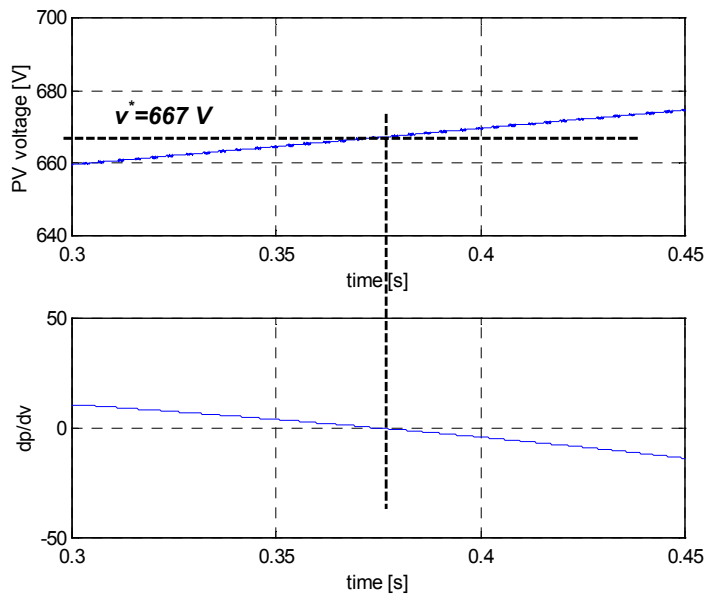

Fig. 9. PV panel voltage and computed power derivative $\partial p / \partial v$ around the maximum power operating point

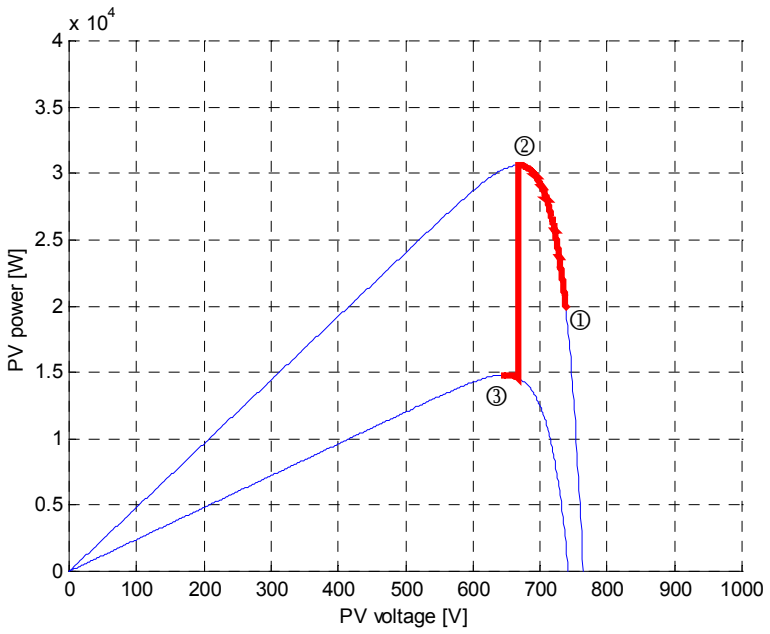

Fig. 11. Effect of a $-50 \%$ solar irradiance transient on the $P-V$ diagram 

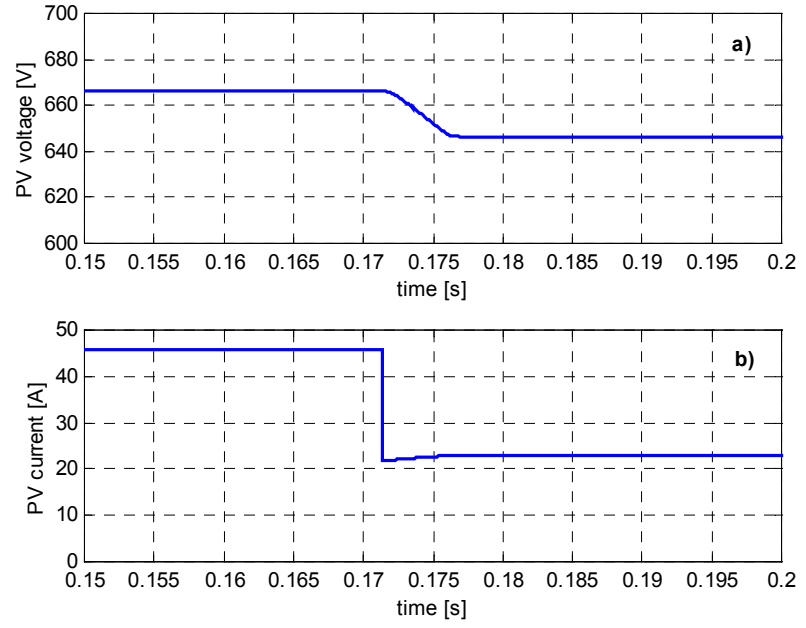

Fig. 12. Effect of a $-50 \%$ solar irradiance transient a) PV voltage vs. time

b) PV current vs. time
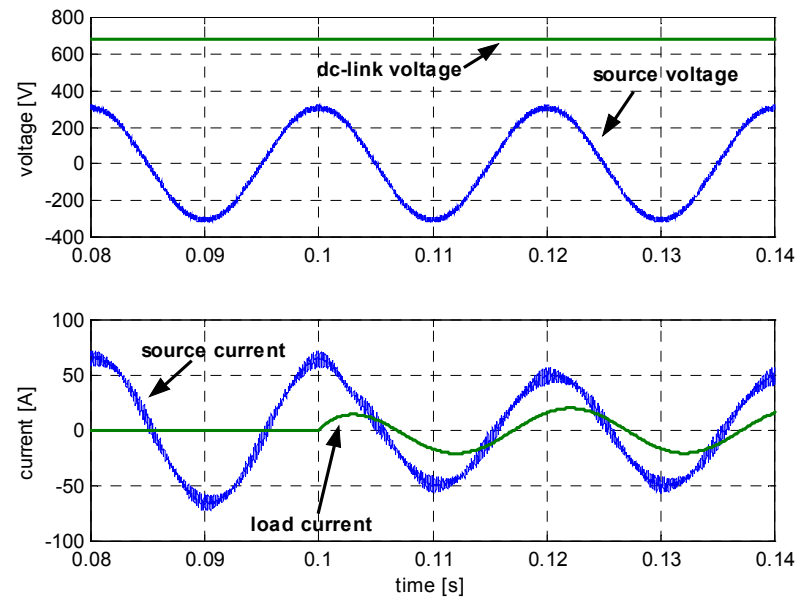

Fig. 14. Effect of a load switching-on

a) PV voltage and source voltage

b) source current and load current

Fig. 14 shows the behavior of the system during the switching-on of a load $\left(P_{L}=7.5 \mathrm{~kW}, \cos \varphi=0.8\right)$. After the load insertion, the source current $i_{S A}$ rapidly changes its amplitude and remains sinusoidal and in phase agreement with the corresponding line to neutral source voltage.

\section{CONCLUSION}

The relatively low voltage excursion of the maximum power point corresponding to different environmental conditions, make it possible to consider the effectiveness a direct coupling of PV panels and grid inverter. This simplified configuration avoids the use of an intermediate dc chopper, reducing the losses and improving the reliability of the whole system. In order to maximize the conversion efficiency, an improved MPPT algorithm is proposed in this paper, based on an analogue processing of current and voltage ripple of the PV panels caused by the inverter PWM switching operation. The MPPT algorithm is based on the regulation of the dc-link voltage within a safety excursion range. The PWM-VSI acts as a power active filter, contributing to the power quality requirement of the utility grid. A complete set of realistic numerical

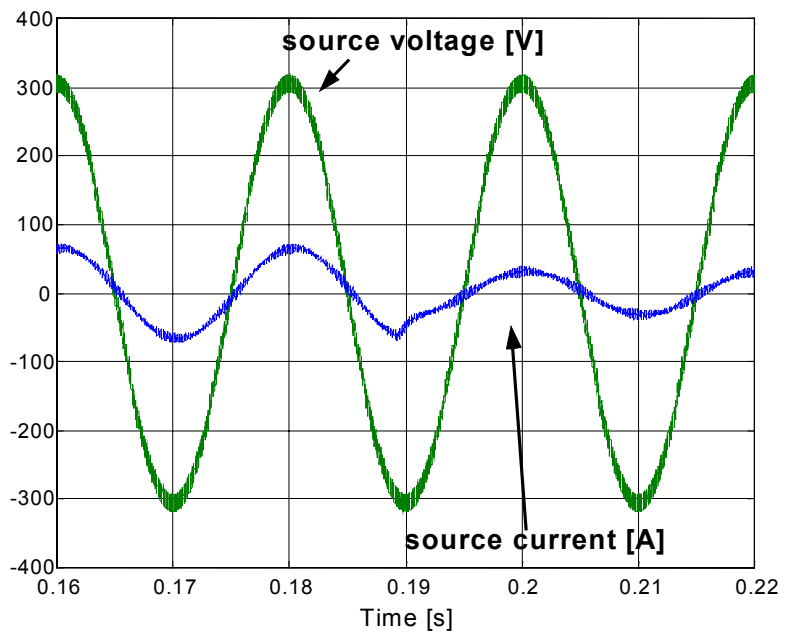

Fig. 13. Effect of a $-50 \%$ solar irradiance transient on the source current

simulations has been presented in the paper, with reference to steady-state and dynamic operating conditions. The results show the good performance of the control system and confirm the effectiveness of the proposed PV generation system.

\section{REFERENCES}

[1] D. Casadei, G. Grandi, U. Reggiani, G. Serra, "Analysis of a Power Conditioning System for Superconducting Magnetic Energy Storage," Proc. of IEEE International Symposium on Industrial Electronics, ISIE, Pretoria (SA), July 7-10, 1998

[2] D. Casadei, G. Grandi, C. Rossi, "Effects of Supply Voltage non-Idealities on the Behavior of an Active Power Conditioner for Cogeneration Systems," Proc. of IEEE Power Electronic Specialist Conference, PESC, Galway (Ireland), 18-23 June, 2000

[3] Y.C. Kuo, T.J. Liang, J.F. Chen, "Novel maximum-power-point-tracking controller for photovoltaic energy conversion system," IEEE Trans. on Industrial Electronics, Vol. 48 No. 3, June 2001, pp. 594-601

[4] T.Y. Kim, H.G. Ahn, S.K. Park, Y.K. Lee, "A novel maximum power point tracking control for photovoltaic power system under rapidly changing solar radiation," Proc. of IEEE International Symposium on Industrial Electronics, ISIE 2001, Pusan, Korea, Vol. 2, pp. 1011-1014

[5] J.A. Gow, C.D. Manning, "Controller arrangement for boost converter systems sourced from solar photovoltaic arrays or other maximum power sources," IEE Proceedings of Electric Power Applications, Vol. 147, No. 1, Jan. 2000, pp. 15-20

[6] P. Midya, P.T. Krein, R.J. Turnbull, R. Reppa, J. Kimball, "Dynamic maximum power point tracker for photovoltaic applications," Proc. of $27^{\text {th }}$ Annual IEEE Power Electronics Specialists Conference, PESC 1996, Vol. 2, pp. 1710-1716

[7] A. Brambilla, M. Gambarara, A. Garutti, F. Ronchi, "New approach to photovoltaic arrays maximum power point tracking," Proc. of $30^{\text {th }} \mathrm{An}$ nual IEEE Power Electronics Specialists Conference, PESC 1999, Vol. 2, pp. 632-637

[8] D.L. Logue, P.T. Krein, "Optimization of power electronic systems using ripple correlation control: a dynamic programming approach," Proc. of IEEE $32^{\text {nd }}$ Annual Power Electronics Specialists Conference, PESC 2001, Vol. 2, pp. 459-464

[9] Y.H. Lim, D.C. Hamill, "Synthesis, simulation and experimental verification of a maximum power point tracker from nonlinear dynamics," Proc. of IEEE 32 nd Annual Power Electronics Specialists Conference, PESC 2001, Vol. 1, pp. 199-204

[10] M.A. de Blas, J.L. Torres, E. Prieto, A. García: "Selecting a suitable model for characterizing photovoltaic devices," Renewable Energy Journal, Vol. 25, No. 3, March 2002, pp. 371-3800.5 